(C) 2020 Universidad Nacional Autónoma de México, Facultad de Estudios Superiores Zaragoza.

Este es un artículo Open Access bajo la licencia CC BY-NC-ND (http://creativecommons.org/licenses/by-nc-nd/4.0/).

TIP Revista Especializada en Ciencias Químico-Biológicas, 23: 1-9, 2020.

https://doi.org/10.22201/fesz.23958723e.2020.0.248

\title{
Identificación de microorganismos aislados de suelos agrícolas con capacidad de tolerar 2.4-D y malatión
}

\author{
María Fernanda Rosado-Flores, Juan Manuel González-Prieto†, \\ Maribel Mireles-Martínez, Jorge Alberto Torres-Ortega, \\ Ninfa María Rosas-García y Jesús Manuel Villegas-Mendoza* \\ Laboratorio de Biotecnología Ambiental, Centro de Biotecnología Genómica, \\ Instituto Politécnico Nacional, Blvd. del Maestro Narciso Mendoza s/n, \\ Reynosa 88710, Tamaulipas, México.*E-mail: jmvillegas@ipn.mx
}

\begin{abstract}
RESUMEN
En el presente estudio, se analizó la diversidad microbiana de los suelos agrícolas de las localidades de Río Bravo y la Estación Cuauhtémoc en el estado de Tamaulipas y en los límites de Dolores Hidalgo en el estado de Guanajuato. Los plaguicidas utilizados fueron 2,4-D y malatión, con los que se establecieron pruebas preselectivas para el aislamiento de los microorganismos y su tolerancia. Para la identificación de las bacterias se amplificó el gen $16 \mathrm{~S}$ y para los hongos la región ITS. El género bacteriano Pseudomonas, así como el género fúngico Penicillium fueron los de mayor abundancia en las muestras analizadas. Los resultados indicaron que las cepas tolerantes fueron Pseudomonas aeruginosa, Stenotrophomonas pavanii y Acinetobacter lactucae creciendo a una concentración $>2.0 \mathrm{~g} \mathrm{~L}^{-1} \mathrm{de}$ 2,4-D y $>1.0 \mathrm{~g} \mathrm{~L}^{-1}$ de malatión. Así como Fusarium sp., a $2.0 \mathrm{~g} \mathrm{~L}^{-1}$ de malatión y $0.9 \mathrm{~g} \mathrm{~L}^{-1}$ de 2,4-D y el hongo Talaromyces variabilis con un crecimiento a $3.1 \mathrm{~g} \mathrm{~L}^{-1}$ de malatión. Para el caso de $S$. pavanii, A. Lactucae y $T$. variabilis no existen reportes de tolerancia a los plaguicidas mencionados, sin embargo, en este trabajo se demuestra por primera vez que pueden ser utilizados en técnicas de biorremediación de suelos.
\end{abstract}

Palabras clave: 2,4-D, malatión, biorremediación, tolerancia, diversidad microbiana.

Identification of microorganisms isolated from agricultural soils

with the capacity to tolerate 2.4-D and malation

\begin{abstract}
In the present study, the microbial diversity was analyzed in agricultural soils from Río Bravo city and Cuauhtémoc Station, Tamp., and the surroundings of the city of Dolores Hidalgo, Guanajuato. Microorganisms isolation and tolerance tests were conducted preselectively using 2,4-D and malathion. Microbial identification was conducted by $16 \mathrm{~S}$ gen amplification in bacteria and ITS region in fungi. The bacterial genus Pseudomonas, and the fungal genus Penicillium were the most abundant in the analyzed samples. Pseudomonas aeruginosa, Stenotrophomonas pavanii and Acinetobacter lactucae grew at a concentration $>2.0 \mathrm{~g} \mathrm{~L}^{-1}$ of 2,4-D and $>1.0 \mathrm{~g} \mathrm{~L}^{-1}$ of malathion. Fusarium sp. grew at $2.0 \mathrm{~g} \mathrm{~L}^{-1}$ of malathion and $0.9 \mathrm{~g} \mathrm{~L}^{-1}$ of 2,4-D herbicide, and Talaromyces variabilis tolerated up to $3.1 \mathrm{~g} \mathrm{~L}^{-1}$ of malathion. There are no reports of tolerance for S. pavanii, A. Lactucae, and T. variabilis to these pesticides so far. Therefore, the semicroorganisms may have the potential to be used in soil bioremediation techniques.
\end{abstract}

Keywords: 2,4-D, malatión, bioremediation, tolerance, microbial diversity. 


\section{INTRODUCCIÓN}

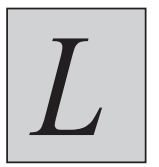

a biorremediación es la eliminación, transformación o atenuación de xenobióticos mediante procesos biológicos llevados a cabo por organismos o los productos de estos (Shukla, Singh \& Sharma, 2010; Adams, Fufeyin, Okoro \& Ehinomen, 2015). Las técnicas de biorremediación pueden ser de dos tipos: la bioestimulación, donde se establecen las condiciones fisiológicas y de nutrición adecuadas, para inducir el crecimiento de los microorganismos nativos y la bioaumentación en la que se utilizan microorganismos específicos, cultivados previamente en presencia de contaminantes (Maheshwari et al., 2014).

Los microorganismos se caracterizan por ejercer una función importante en la degradación de xenobióticos, influir en los procesos físicos o químicos de la degradación y persistir a los compuestos recalcitrantes (Chakrabarty, 2017). Actualmente, las bacterias son las más estudiadas, debido a que cuentan con una extensa capacidad de adaptación (Biswas, 2015), son de crecimiento rápido y en general, más fáciles de cultivar en el laboratorio que otros microorganismos (Ortiz-Hernández, Sánchez-Salinas, Olvera-Velona \& Folch-Mallol, 2011), como las siguientes: Flavobacterium sp., (Sethunathan \& Yoshida, 1973), Sphingomomas sp., (Yao et al, 2015), Actinobacteria, Streptomyces sp., (Wang et al, 2017), Diferentes especies del género Bacillus (Ishag et al, 2017), Stenotrophomonas y Pseudomonas (Deng et al, 2015).

Los hongos, también presentan la capacidad de formar redes miceliales, además de usar el contaminante como sustrato de crecimiento y un sistema enzimático inespecífico, que los convierten en importantes herramientas biológicas para utilizar en la biorremediación (Harms, Schlosser \& Wick, 2011). Como en Phanerochaete sordida (Mori et al., 2017) y los hongos ectomicorrícicos: Boletus edulis, Gomphidius viscidus, Laccaria bicolor, y Leccinum scabrum (Huang et al., 2007). El objetivo de este trabajo fue, a partir de suelos agrícolas mexicanos contaminados con agroquímicos, aislar e identificar microorganismos tolerantes a los plaguicidas, capacidad que los convierte en biodegradadores.

\section{MATERIALES Y MÉTODOS}

Muestreo de suelo agrícola

Se obtuvieron muestras de los estados de Tamaulipas y Guanajuato, del primero en la localidad de la Estación
Cuauhtémoc [ $22^{\circ} 20^{\prime}$ y $22^{\circ} 49^{\prime} \mathrm{N}$; $98^{\circ} 21^{\prime}$ y $97^{\circ} 50^{\prime} \mathrm{O}$; entre 50 y $300 \mathrm{msnm}$ ] y en el municipio de Río Bravo [26 $05^{\circ}$ y $25^{\circ} 23^{\prime}$ $\mathrm{N}$; $98^{\circ} 11^{\prime}$ y $97^{\circ} 51^{\prime} \mathrm{O}$; entre 50 y $500 \mathrm{msnm}$ ] y del segundo en los límites de Dolores Hidalgo [ $21^{\circ} 21^{\prime}$ y $20^{\circ} 51^{\prime} \mathrm{N}$; $100^{\circ} 38^{\prime}$ y $101^{\circ} 13^{\prime} \mathrm{O}$, entre 1,800 y $2,800 \mathrm{msnm}$ ]. Las muestras se tomaron dentro de una parcela, en donde a partir de su centro y hacia el perímetro se eligieron al azar diez sitios en los que de cada uno, y a una profundidad de $10 \mathrm{~cm}$, se extrajo $1 \mathrm{Kg}$ de tierra, completando un total de $10 \mathrm{Kg}$ que se secaron a temperatura ambiente durante $24 \mathrm{~h}$, posteriormente se tamizaron en una malla del número 2 con una apertura de $20 \mathrm{~mm}$, para obtener una muestra fina y homogénea. Las muestras se almacenaron en bolsas estériles a $4{ }^{\circ} \mathrm{C}$.

\section{Criterio de selección para la concentración}

Las concentraciones de 2,4 D y Malatión en la primera preselección son las mínimas reportadas para cada plaguicida en la literatura y en la segunda pre-selección se utilizaron las concentraciones máximas reportadas tanto para bacterias como hongos. (Tabla I)

\section{Cultivo preselectivo de bacterias}

La primer pre-selección se realizó en matraces de $250 \mathrm{~mL}$, que contenían $100 \mathrm{~mL}$ de medio mínimo (MM), adicionado con $10 \mathrm{~g}$ de tierra seca tamizada, y como única fuente de carbono se agregó la concentración mínima del plaguicida; para inhibir el crecimiento fúngico se añadió $9.4 \mu \mathrm{L}^{-1}$ de carboxina [16 $\mu \mathrm{M}$ ], incubándose durante $48 \mathrm{~h}$ a $37{ }^{\circ} \mathrm{C}$ y $170 \mathrm{rpm}$ (Thermo Scientific, 435, MAXQ 480R HP). El control positivo consistió en un matraz sin plaguicida, con $1 \mathrm{~g}$ de glucosa como fuente de carbono y un control negativo sin glucosa. Transcurrido el periodo de incubación, se separó la fase acuosa del sedimento, filtrando el tratamiento con ayuda de un papel filtro de 10 x $10 \mathrm{~cm}$ y un embudo estéril, el sobrenadante se almacenó a $4^{\circ} \mathrm{C}$ hasta su uso.

En la segunda preselección se utilizaron tubos Falcón estériles de $50 \mathrm{~mL}$, a los que se añadieron $20 \mathrm{~mL}$ de medio mínimo (MM), 1 mLdel filtrado obtenido y las concentraciones máximas reportadas de los plaguicidas; con las mismas condiciones de incubación ya mencionadas. Al término del periodo de incubación se realizaron diluciones seriadas con agua estéril $\left(10^{-1}\right.$ a $\left.10^{-4}\right)$ de cada una de las concentraciones. Después se inocularon $500 \mu \mathrm{L}$ (de cada dilución) en el centro de una caja de Petri de 100 x 15 mm con Agar y Medio Mínimo (adicionado

Tabla I. Concentraciones ensayadas en bacterias y hongos de los plaguicidas seleccionados.

\begin{tabular}{|c|c|c|c|}
\hline Plaguicida & Concentración mínima & $\begin{array}{c}\text { Concentración máxima } \\
\text { en bacterias }\end{array}$ & $\begin{array}{c}\text { Concentración } \\
\text { máxima en hongos }\end{array}$ \\
\hline $\begin{array}{c}2,4 \text { - diclorofenoxiacético } \\
(2,4-\mathrm{D})\end{array}$ & $100 \mathrm{mg} \mathrm{L}^{-1}$ & $2500 \mathrm{mg} \mathrm{L}^{-1}$ & $2500 \mathrm{mg} \mathrm{L}^{-1}$ \\
\hline Malatión & $50 \mathrm{mg} \mathrm{L}^{-1}$ & $500 \mathrm{mg} \mathrm{L}^{-1}$ & $400 \mathrm{mg} \mathrm{L}^{-1}$ \\
\hline
\end{tabular}


con la concentración del plaguicida correspondiente, el inóculo se distribuyó en toda la superficie del agar con perlas de vidrio estériles y se incubó durante $24 \mathrm{~h}$ a $37^{\circ} \mathrm{C}$, o hasta observar el crecimiento bacteriano ( $\mathrm{CO}_{2}$ Incubator, Shel LAB). Después se aislaron las bacterias tomando una porción de la colonia con un asa bacteriológica estéril y se sembró mediante estriado cruzado en cajas de Petri de 100 x 15 con Agar Nutritivo (AN) (adicionado con $9.4 \mu 1 \mathrm{~L}^{-1}$ de carboxina) marca MCD LAB., incubándose durante $12 \mathrm{~h}$ a $37^{\circ} \mathrm{C}$. Las cajas se almacenaron a $4{ }^{\circ} \mathrm{C}$ hasta su uso.

\section{Cultivo preselectivo de hongos}

El cultivo de hongos es el mismo que para las bacterias con las siguientes variables: el periodo de incubación fue de $72 \mathrm{ha} 28^{\circ} \mathrm{C}$ y $250 \mathrm{rpm}$, para la inhibición de las bacterias se adicionó $1 \mu \mathrm{L}$ $\mathrm{mL}^{-1}$ de ampicilina $\left[100 \mu \mathrm{g} \mathrm{mL}^{-1}\right.$. En la segunda preselección para la incubación de las colonias también se utilizaron cajas Petri $(100 \times 15 \mathrm{~mm})$ en un período que puede variar hasta observar el crecimiento fúngico de 4 días a $28^{\circ} \mathrm{C}$. El aislamiento de los hongos se realizó con un palillo estéril tomando una porción del micelio que se colocó, por picadura, en el centro del Agar Papa Dextrosa (PDA) marca MCD LAB, contenido en una caja Petri de $(100 \times 15 \mathrm{~mm})$, para incubación durante $72 \mathrm{~h}$ a $28^{\circ} \mathrm{C}$.

\section{Morfología de los microorganismos aislados}

La identificación de las colonias bacterianas se realizó utilizando el manual Bergey's of Systematic Bacteriology (Brenner et al, 2005), posteriormente en un portaobjetos se fijaron por calor y tiñeron utilizando la tinción de Gram para observarlas con un microscopio óptico tri ocular (Leica DM750, Leica ICC50 HD) utilizando el objetivo $100 \mathrm{X}$ (objetivo de inmersión) empleando el Software Las EZ v. 3.0.0 Copyright $(C) 2013$ Leica Microsystems. Las colonias-bacterianas se agruparon de acuerdo con sus diferencias fenotípicas y su morfología celular.

Para la identificación macro-morfológica fúngica se realizaron cultivos monospóricos; tomando en cuenta las características fenotípicas presentadas en el anverso (tamaño, color y forma) y reverso (pigmento) de la colonia. La micro-morfología (tipo de micelio y espora, modalidad de micelio, morfología de esporas, forma de vesícula y conidios) se identificó realizando la técnica de microcultivo. El montaje se visualizó en un microscopio óptico tri ocular usando los objetivos 40 X y $100 \mathrm{X}$.

\section{Identificación molecular}

Para la identificación de las cepas bacterianas, se realizó la extracción de DNAg mediante el protocolo comercial de Wizard ${ }^{\circledR}$ Genomic DNA Purification Kit (Promega, A1120), de acuerdo a las instrucciones del fabricante. Posteriormente, se amplificó el gen 16S rDNA usando los oligonucleótidos universales dD1 (5'- AGAGTTTGATCCTGGCTCAG -3') y rP2 (5' - ACGGCTACCTTGTTACGACTT -3') diseñados por Weisburg (1991) y sintetizados por Biosynthesis (Lewisville, $\mathrm{Tx})$. La reacción de amplificación contenía $12.5 \mu \mathrm{l}$ de PCR
PCRGoTaq ${ }^{\circledR}$ Master Mix 2 X (Promega ${ }^{\mathrm{TM}}$ ), $1 \mu \mathrm{L}$ de cada oligonucleótido $(5 \mu \mathrm{M})$ y $1 \mu \mathrm{L}$ de DNAg $(50 \mathrm{ng})$ en un volumen final de $25 \mu \mathrm{L}$. Las condiciones de PCR usadas fueron un alineamiento a $94{ }^{\circ} \mathrm{C} / 5 \mathrm{~min}$; seguido de 35 ciclos de $94{ }^{\circ} \mathrm{C} / 85 \mathrm{~s}, 58{ }^{\circ} \mathrm{C} / 90 \mathrm{~s}, 72{ }^{\circ} \mathrm{C} / 60 \mathrm{~s}$; una extensión final a $72{ }^{\circ} \mathrm{C} / 7 \mathrm{~min}$. Después del gel de electroforesis, el producto de PCR fue purificado con ExoSAP-IT Clean up (Affly metrix, Inc) y cuantificado. Posteriormente fue enviado a Eurofins Genomics (EUA) de acuerdo a las instrucciones del proveedor. Las secuencias obtenidas fueron analizadas en el GenBank usando la base de datos BLAST.

Para la extracción de DNAg fúngico, se utilizó el método de cloroformo:octanol (24:1) de Reader \& Broda (1985). Para ello se partió de $1 \mathrm{~mL}$ de cultivo de $48 \mathrm{~h}$ de crecimiento, al finalizar el DNA obtenido se cuantificó por Nano Drop, después, se amplificó la región ITS, utilizado los oligonucleótidos universales ITS1 (5'-TCCGTAGGTGAACCTGCGG-3')e ITS4 (5'- TCCTCCGCTTATTGATATGC - 273') (White, Bruns, Lee \& Taylor, 1990) sintetizados por Biosynthesis (Lewisville, Tx). La reacción de amplificación fue la misma que en el protocolo anterior. Las condiciones de PCR usadas fueron un alineamiento a $94{ }^{\circ} \mathrm{C} / 5 \mathrm{~min} ; 35$ ciclos de $94{ }^{\circ} \mathrm{C} / 30 \mathrm{~s}, 58^{\circ} \mathrm{C} / 30 \mathrm{~s}, 72^{\circ} \mathrm{C} / 60 \mathrm{~s}$; una extensión final a $72^{\circ} \mathrm{C} / 7 \mathrm{~min}$. Después del gel de electroforesis el producto fue purificado, secuenciado y analizado de acuerdo al protocolo anterior.

\section{Pruebas de tolerancia en bacterias}

En tubos de fondo plano $(2.3 \times 9.5 \mathrm{~cm})$, se añadieron $15 \mathrm{~mL}$ de caldo nutritivo y se adicionaron las concentraciones de los plaguicidas y el inóculo $\left(1 \times 10^{8}\right.$ células $\left.\mathrm{mL}^{-1}\right)$; se incubó durante $24 \mathrm{~h}$ a $37^{\circ}$ y $170 \mathrm{rpm}$. Las concentraciones utilizadas para el herbicida 2 ,4-D fueron de $1.0 ; 1.25 ; 1.50 ; 1.75 ; 2.0$; $2.5 ; 3.0$ y $3.5 \mathrm{~g} \mathrm{~L}^{-1}$. Para el insecticida malatión se utilizaron concentraciones a partir de $0.5 ; 0.10 ; 0.15 ; 0.20 ; 0.25 ; 0.50$; $1.0 ; 1.25 ; 1.50$ y $2.0 \mathrm{~g} \mathrm{~L}^{-1}$. La tolerancia se midió con base en la concentración celular (crecimiento microbiológico), el cual se determinó por las variantes de densidad óptica (DO), la lectura se analizó en un espectrofotómetro (Cintra 10e, UVVisible Spectrometer, GBC), utilizando el Software Cintral v 2.4 a una longitud de onda de $625 \mathrm{~nm}$. El control negativo se efectuó en un medio enriquecido sin inóculo ni plaguicida, para el control positivo se utilizó el mismo medio inoculado con el microorganismo, sin plaguicida. Los intervalos de medición establecidos incluían la medición inicial $(0 \mathrm{~h})$ y a las $24 \mathrm{~h}$ posteriores a la inoculación todos los tratamientos se efectuaron por triplicado.

\section{Pruebas de tolerancia en hongos}

Estas pruebas, se ejecutaron bajo el mismo procedimiento que en las pruebas de tolerancia bacterianas, modificando el medio a caldo papa dextrosa y el periodo de incubación de $72 \mathrm{~h}$ a $28^{\circ} \mathrm{C}, 250 \mathrm{rpm}$. Las concentraciones para el herbicida 2,4-D se establecieron dentro de un intervalo de 0.03 ; 0.06 ; 
$0.12 ; 0.25 ; 0.50 ; 1.0$ y $2.5 \mathrm{~g} \mathrm{~L}^{-1}$ y para el insecticida malatión se realizaron concentraciones de $0.01 ; 0.03 ; 0.06 ; 0.12 ; 0.25 ; 0.50$; $1.0 ; 1.5 ; 2.0 ; 2.5 ; 2.7$ y $3.0 \mathrm{~g} \mathrm{~L}^{-1}$. Los intervalos de medición se establecieron a las $0,24,48$ y $72 \mathrm{~h}$ posteriores a la inoculación.

\section{Análisis estadístico}

Para determinar la tolerancia de los microorganismos a los plaguicidas se utilizó el programa estadístico IBM SPSS Statistics versión 22 utilizando el análisis de regresión, ejecutando el modelo Probit con prueba de significancia estadística $\mathrm{P}>0.05$.

\section{RESUltados}

Se aislaron 68 bacterias y 6 hongos de la localidad Estación Cuauhtémoc, mientras que en la de Río Bravo fueron 90 bacterias y 12 hongos, por último del estado de Guanajuato se aislaron 120 bacterias y 3 hongos. Para la identificación molecular de las 278 bacterias se agruparon según su similitud morfológica y de esos grupos se escogieron 18 aislados representativos. En lo que concierne a los 21 hongos se seleccionaron 16 aislados representativos (Tabla II).

En el análisis molecular de los aislados de bacterias se identificaron: Pseudomonas plecoglossicida, Acinetobacter lactucae, Pseudomonas aeruginosa, Acinetobacter pittii y Stenotrophomonas pavanii (Tabla III).

Para el caso de los hongos se identificaron los géneros Penicillium, Talaromyces, Aspergillus, Fusarium, Cladosporium y Rasamsonia. (Tabla IV).
Los microorganismos que crecieron en las concentraciones más altas de los plaguicidas, evaluados, 2,4-D y malatión, se infirieron como tolerantes. Las bacterias Acinetobacter lactucae, Pseudomonas aeruginosa y Stenotrophomonas pavanii mostraron la mayor tolerancia al herbicida 2,4-D a una concentración $>2.0 \mathrm{~g} \mathrm{~L}^{-1} \mathrm{y}>1.0 \mathrm{~g} \mathrm{~L}^{-1}$ al insecticida malatión, con excepción de la bacteria $A$. lactucae que, con

Tabla II. Selección de aislados bacterianos y fúngicos.

\begin{tabular}{|l|c|c|}
\hline \multicolumn{1}{|c|}{ Claves } & Bacterias & Hongos \\
\hline $\mathrm{B}=$ Bacteria & BDE13 & HDR1 \\
$\mathrm{H}=$ Hongo & BMaE2 & HMaG4 \\
$\mathrm{D}=2.3 \mathrm{D}$ & BMaE1 & HMaR7 \\
$\mathrm{Ma}=$ Malatión & BDR22 & HDE1 \\
$\mathrm{E}=$ Estación & BDR11 & HDE5 \\
Cuauhtémoc & BDR28 & HMaR8 \\
$\mathrm{R}=$ Río Bravo & BMaR1 & HMaR4 \\
$\mathrm{G}=$ Guanajuato & BMaR3 & HDG2 \\
$\#=$ Número de & BMaR5 & HDG6 \\
aislado & BMaR16 & HMaE1 \\
& BDG15 & HMaE2 \\
& BDG18 & HMaE3 \\
& BDG17 & HMaR2 \\
& BDG8 & HMaR5 \\
& BDG36 & HMaR6 \\
& BDG35 & HMaE8 \\
& BMaG1 & \\
\hline
\end{tabular}

Tabla III. Análisis molecular de los aislados bacterianos.

\begin{tabular}{|c|l|c|c|}
\hline Clave & \multicolumn{1}{|c|}{ Cepa } & \% identidad & Número de acceso \\
\hline BDE13 & Pseudomonas aeruginosa cepa DSM 50071 & 98.50 & NR_117678.1 \\
\hline BDR11 & Acinetobacter lactucae cepa NRRL B-41902 & 99.86 & NR_152004.1 \\
\hline BDR22 & Acinetobacter pittii DSM 21653 cepa ATCC 19004 & 96.08 & NR_117621.1 \\
\hline BDR28 & Acinetobacter pittii DSM 21653 cepa ATCC 19004 & 99.79 & NR_117621.1 \\
\hline BDG8 & Pseudomonas plecoglossicida cepa NBRC 103162 & 99.64 & NR_114226.1 \\
\hline BDG15 & Pseudomonas plecoglossicida cepa NBRC 103162 & 99.64 & NR_114226.1 \\
\hline BDG17 & Pseudomonas plecoglossicida cepa NBRC 103162 & 99.19 & NR_114226.1 \\
\hline BDG35 & Acinetobacter lactucae cepa NRRL B-41902 & 99.01 & NR_152004.1 \\
\hline BMaE1 & Atlantibacter hermannii cepa CIP 103176 (Escherichia hermannii) & 97.88 & NR_104940.1 \\
\hline BMaE2 & Acinetobacter pittii DSM 21653 cepa ATCC 19004 & 99.36 & NR_117621.1 \\
\hline BMaR1 & Pseudomonas aeruginosa cepa DSM 50071 & 99.06 & NR_117678.1 \\
\hline BMaR3 & Stenotrophomonas pavanii cepa LMG 25348 & 99.50 & NR_118008.1 \\
\hline BMaR5 & Pseudomonas plecoglossicida cepa NBRC 103162 & 99.22 & NR_114226.1 \\
\hline BMaR16 & Pseudomonas plecoglossicida cepa NBRC 103162 & 99.00 & NR_114226.1 \\
\hline BMaG1 & Pseudomonas aeruginosa cepa DSM 50071 & 98.13 & NR_117678.1 \\
\hline BMaG3 & Pseudomonas plecoglossicida cepa NBRC 103162 & 99.64 & NR_114226.1 \\
\hline
\end{tabular}


Tabla IV. Análisis molecular de los aislados fúngicos.

\begin{tabular}{|c|l|c|c|}
\hline Clave & \multicolumn{1}{|c|}{ Cepa } & \% Identidad & Número de acceso \\
\hline HDE1 & Aspergillus sp. isolate FS-1 & 98.61 & MK817589.1 \\
\hline HDE5 & $\begin{array}{l}\text { Talaromyces variabilis strain NRRL2125 } \\
\text { (Penicillium variabile) }\end{array}$ & 98.02 & KF984797.1 \\
\hline HMaE1 & Cladosporium sp. aislado UoMD16-15 & 99.57 & MF967429.1 \\
\hline HMaE2 & Penicillium citrinum strain PcN25A01 & 99.34 & MK852473.1 \\
\hline HMaE3 & Aspergillus ustus cepa NRRL 1974 & 99.45 & AY373879.1 \\
\hline HDR1 & Fusarium sp. Isolate DS921 & 99.14 & MK809003.1 \\
\hline HMaR7 & Penicillium citrinum strain PcN25A01 & 99.77 & MK852473.1 \\
\hline HMaR8 & Penicillium citrinum strain PcN25A01 & 99.55 & MK852473.1 \\
\hline HMaR4 & Talaromyces variabilis strain NRRL2125 & 99.03 & MK984797.1 \\
\hline HMaR2 & Penicillium singorense isolate ST016 & 99.38 & MK817632.1 \\
\hline HMaR5 & Penicillium sp. isolate FL-18 & 100 & KX953540.1 \\
\hline HMaR6 & Penicillium sp. strain RRA89 & 99.59 & MK852473.1 \\
\hline HDG2 & Penicillium citrinum strain PcN25A01 & 96.90 & MK271294.1 \\
\hline HDG6 & Talaromyce sfavus strain Z2 & 94.46 & MK852473.1 \\
\hline HMaG4 & Penicillium citrinum strain PcN25A01 & 100 & \\
\hline
\end{tabular}

este último plaguicida fue ligeramente menor en comparación con las concentraciones toleradas por Acinetobacter pittii (Tabla V).

De acuerdo con el análisis estadístico, la mayor tolerancia en los hongos se <observó en Fusarium sp., que creció a una concentración $>0.9 \mathrm{~g} \mathrm{~L}^{-1}$ con el herbicida 2,4-D y $>2.0 \mathrm{~g} \mathrm{~L}^{-1}$ con el insecticida malatión, para este último compuesto T. variabilis toleró $>3.0 \mathrm{~g} \mathrm{~L}^{-1}$ (Tabla VI).

\section{Discusión}

Las técnicas de biorremediación son importantes para desintoxicar sitios contaminados por xenobióticos recalcitrantes, como algunos agroquímicos. En este trabajo, los plaguicidas 2,4-D y malatión, fueron seleccionados por su amplia aplicación en los suelos de las regiones agrícolas muestreadas, además, son considerados tóxicos para el ser humano. Asimismo, en los microorganismos identificados se evaluó la tolerancia a esos plaguicidas. La bacteria Pseudomonas aeruginosa se eligió como tolerante, ya que mostró crecimiento a las concentraciones más altas evaluadas en este estudio, que fueron de 2.9 y $1.3 \mathrm{~g} \mathrm{~L}^{-1}$ de $2,4-\mathrm{D}$ y malatión, respectivamente. Shafiani \& Malik (2003), aislaron e identificaron al género Pseudomonas a partir de suelo agrícola, la bacteria se consideró como tolerante al plaguicida malatión a una concentración de $1,600 \mu \mathrm{g} \mathrm{mL}^{-1}$. Estudios realizados por Abo-Amer (2007), reportaron que P. aeruginosa presentó la capacidad de degradar $42.5 \mathrm{mg} \mathrm{L}^{-1}$ del insecticida malatión en un medio adicionado con peptona y extracto de levadura, el compuesto actúa como fuente secundaria de carbono, así como
Saafan, Azmy, Amin, Ahmed \& Essam (2016), registraron hallazgos similares con esta bacteria, la cual mostró el potencial de degradar hasta el $89 \%$ del malatión a partir de $100 \mathrm{mg} \mathrm{L}^{-1}$ adicionado con extracto de levadura y glucosa con los que visualizaron un aumento en la biomasa microbiana.

Tabla V. Tolerancia en bacterias.

\begin{tabular}{|l|c|c|}
\hline \multicolumn{1}{|c|}{ Bacteria } & $\begin{array}{c}\mathbf{D L}_{\mathbf{5 0}} \\
\mathbf{2 , 4 - D g r / l}\end{array}$ & $\begin{array}{c}\mathbf{D L}_{\mathbf{5 0}} \\
\text { malation }_{\text {gr/l}}\end{array}$ \\
\hline Acinetobacter lactucae & 2.084 & 1.069 \\
\hline Acinetobacter pittii & 1.702 & 1.084 \\
\hline Atlantibacter hermannii & 0.841 & 0.888 \\
\hline Pseudomonas aeruginosa & 2.978 & 1.315 \\
\hline $\begin{array}{l}\text { Pseudomonas } \\
\text { plecoglossicida }\end{array}$ & 1.438 & 1.007 \\
\hline Stenotrophomonas pavanii & 2.462 & 1.200 \\
\hline
\end{tabular}

Tabla VI. Tolerancia en hongos.

\begin{tabular}{|l|c|c|}
\hline \multicolumn{1}{|c|}{ Hongo } & $\begin{array}{c}\mathbf{D L}_{\mathbf{5 0}} \\
\mathbf{2 , 4 - D g r / l}\end{array}$ & $\begin{array}{c}\mathbf{D L}_{\mathbf{5 0}} \\
\text { malatióngr/l }\end{array}$ \\
\hline Aspergillus oryzae & 0.154 & 0.016 \\
\hline Fusarium sudanense & 0.904 & 2.072 \\
\hline Penicillium griseofulvum & 0.234 & 0.108 \\
\hline Rasamsonia sp. & 0.392 & 0.184 \\
\hline Talaromyces variabilis & 0.320 & 3.172 \\
\hline
\end{tabular}


Por otra parte, la bacteria Stenotrophomonas pavanii creció a una concentración de $2.5 \mathrm{~g} \mathrm{~L}^{-1}$ del herbicida 2,4-D y $1.2 \mathrm{~g} \mathrm{~L}^{-1}$ del insecticida malatión, por lo que se seleccionó como tolerante. Sin embargo, $S$. pavanii no ha sido reportada en la tolerancia o degradación a los plaguicidas evaluados en este estudio. No obstante, Feng et al., (2017), reportaron a esta especie con la capacidad de degradar el insecticida organofosforado clorpirifos, utilizado como única fuente de carbono, la bacteria degradó el $90 \%$ del compuesto a partir de una concentración de $5 \mathrm{mg} \mathrm{L}^{-1}$. Además, algunos autores han registrado a especies del género Stenotrophomonas con la capacidad de degradar plaguicidas como el DDT, clorpirifos, fenvalerato, paratión, paraoxón, diazinón y foxim (Chen, Yang, Hu \& Liu, 2011; Dubey \& Fulekar, 2012; Deng et al, 2015; Barragan-Huerta et al, 2007).

La bacteria Acinetobacter lactucae, igualmente se seleccionó como tolerante debido a que creció a una concentración de 2.0 y $1.0, \mathrm{~g} \mathrm{~L}^{-1}$ del $2,4-\mathrm{D}$ y malatión, respectivamente. Cabe mencionar que esa bacteria no ha sido estudiada en la degradación de los plaguicidas 2,4-D y malatión. Sin embargo, el género Acinetobacter ha sido reportado con la capacidad de degradar al plaguicida atrazina en medio mínimo, utilizado como la única fuente de carbono (Mirgain, Green \& Monteil, 1993; Singh, Suri \& Cameotra, 2004).

Por otra parte, en el hongo Cladosporium sp., no se logró medir la tolerancia mediante métodos turbidimétricos, debido al tipo de desarrollo que presentó, por lo que, es viable realizar la evaluación utilizando una metodología diferente como lo es la medición del crecimiento radial de la colonia (Arshad \&Aishatul, 2015). Elhongo Fusarium sp., se eligió como tolerante por presentar un crecimiento en concentraciones de 0.9 y $2.0 \mathrm{~g} \mathrm{~L}^{-1}$ del 2,4-D y malatión, respectivamente. Peter, Gajendiran, Mani, Nagaraj \& Abraham (2015), registraron a la especie Fusarium oxysporum con la capacidad de degradar completamente al plaguicida malatión a una concentración de $400 \mathrm{mg} \mathrm{L}^{-1}$, este fue adicionado como única fuente de carbono en medio mínimo. Asimismo, este género se ha registrado con la capacidad de degradar otro tipo de compuestos como el poli succinato de butileno (PBS) (Mao, Liu, Gao, Su \& Wang, 2015) e hidrocarburos aromáticos policíclicos (PAHs) (Potin, Rafin \& Veignie, 2004; Wei et al, 2017). El hongo Talaromyces variabilis se seleccionó como tolerante únicamente al plaguicida malatión, porque creció a una concentración de $3.0 \mathrm{~g} \mathrm{~L}^{-1}$. Sin embargo, esta especie no ha sido reportada en la tolerancia o degradación de los plaguicidas evaluados en el presente trabajo. El género Talaromyces se ha caracterizado por incluir especies resistentes al calor (Yamashita, Nakagawa, Sakaguchi,Arima \& Kikoku, 2018) de procesos industriales como la pasteurización, que impacta significativamente a diversos tipos de alimentos (Panek \& Frąc, 2018).

Las bacterias Acinetobacter pitii, Atlantibacter hermanii y Pseudomonas plecoglossicida presentaron un crecimiento $<2.0 \mathrm{~g} \mathrm{~L}^{-1}$ con el herbicida $2,4-\mathrm{D}$ e igual o $<1.0 \mathrm{~g} \mathrm{~L}^{-1}$ con el insecticida malatión, por lo que no se consideraron como tolerantes ni los hongos Aspergillus sp., Penicillium citrinum y Rasamsonia sp., debido a que mostraron un crecimiento a una concentración $<0.4 \mathrm{~g} \mathrm{~L}^{-1}$ en ambos plaguicidas. De los microorganismos mencionados Aspergillus sp., se ha utilizado en evaluaciones de degradación de los plaguicidas arbendazim, captan, mancozeb, metalaxil, tiram y carbofuran (Arshad \& Aishatul, 2015). En el presente trabajo, las bacterias seleccionadas como tolerantes crecieron en concentraciones más altas del plaguicida 2,4-D (2.978 $\left.\mathrm{g} \mathrm{L}^{-1}\right)$ en comparación a las evaluadas en los hongos $\left(0.9 \mathrm{~g} \mathrm{~L}^{-1}\right)$. Lo anterior podría suceder debido a que las bacterias usan al plaguicida como fuente de nutrientes y por ello logran tolerar concentraciones más altas (Mohiddin \& Khan, 2013), en contraste con los hongos, en los que el plaguicida o sus metabolitos podrían permanecer en la biomasa sin presentar transformación(Botero, Mougin, Peñuela \& Barriuso, 2017). La tolerancia a los plaguicidas se relaciona principalmente a la diversidad genética que codifica a enzimas especializadas en la degradación, esto se ve influenciado por los compuestos (Mousawi, 2005; Drouin, Sellami, Prevost, Fortin \& Antoun, 2010), ya que un plaguicida induce a la formación de nuevas vías metabólicas en los microorganismos (Ahemad \& Khan, 2011).

Además, hay autores que reportan especies bacterianas con la capacidad de degradar al herbicida 2,4-D en las que se incluyen a Flavobacterium sp. (Chaudhry \& Huang, 1988), Achromobacter sp. (Xia etal., 2017), F. peregrinum, Arthrobacter sp. (Motosugi \& Soda, 1983), Brevundimonas sp. (Smejkal, Seymour, Burton \& Lappin-Scott, 2003) y especies fúngicas como Phanerochaete chrysosporium (Donnelly, Entry \& Crawford, 1993; Yadav \& Reddy, 1993), Paxillus involutus y Suillus variegatus (Meharg, Cairney \& Maguire, 1997), Umbelopsis isabellina (Nykiel-Szymańska, Stolarek \& Bernat, 2018). Por otro lado, las estrategias de biorremediación en México se han realizado con hongos del género Trichoderma, microorganismos nativos o consorcios para la degradación de plaguicidas como el DDT y sus metabolitos, el insecticida paratión, los herbicidas atrazina y 2,4-D. Lo anterior, se ha realizado en suelos bajo condiciones de laboratorio (Ortiz-Hernández, Monterrosas-Brisson, YanezOcampo \& Sánchez-Salinas, 2001; Robles-González et al., 2006; Islas- Pelcastre et al., 2013; Ortiz, Velasco, Le Borgne \& Revah, 2013). Además, se han llevado a cabo estudios de biodegradación utilizando consorcios bacterianos, en los que se incluyen a las bacterias Stenotrophomonas malthophilia, Proteus vulgaris, Vibrio metschinkouii, Serratia ficaria, Serratia sp. y Yersinia enterocolitica; estos fueron evaluados en la degradación de los insecticidas tetraclorvinfos y paratión (Ortiz-Hernández et al., 2001; Ortiz-Hernández \& Sánchez-Salinas, 2010). A pesar de que en México no se han registrado estudios de biorremediación con la bacteria Acinetobacter lactucae, en el presente trabajo se evidenció su potencial capacidad para tolerar el insecticida malatión y por ésto sea posible su utilización en las técnicas de biorremediación de suelos. 


\section{Conclusiones}

Las cepas con mas tolerancia a 2,4-D y malatión fueron Pseudomonas aeruginosa, Stenotrophomonas pavanii, Acinetobacter lactucae, Fusarium sp. y Talaromyces variabilis. Para el caso de S. pavanii, A. Lactucae y T. variabilis no existen reportes de tolerancia a los plaguicidas mencionados, sin embargo, en este trabajo se demuestra por primera vez que pueden ser utilizados en técnicas de biorremediación de suelos.

\section{Agradecimientos}

Al Instituto Politécnico Nacional y al laboratorio de biotecnología vegetal del Centro de Biotecnología Genómica.

\section{REFERENCIAS}

Abo-Amer,A. (2007). Involvement of chromosomally-encoded genes in malathion utilization by Pseudomonas aeruginosa AA112. Acta microbiologica etimmunologica Hungarica, 54 (3), 261-277. DOI: 10.1556/AMicr.54.2007.3.3.

Adams, G. O., Fufeyin, P. T., Okoro, S. E., \& Ehinomen, I. (2015). Bioremediation, biostimulation and bioaugmention: a review. International Journal of Environmental Bioremediation \& Biodegradation, 3 (1): 28-39. DOI: 10.12691/ijebb-3-1-5.

Ahemad, M. \& Khan, M. S. (2011). Assessment of pesticidetolerance and functional diversity of bacterial strains isolated from rhizospheres of different crops. Insight Microbiol., 1, 8-19. DOI: 10.5567/IMICRO-IK.2011.8.19.

Arshad,A. M.\&Aishatul, B.(2015). Aspergillus niger-a novel heavy metal bio-absorbent and pesticide tolerant fungus. Res. J. Chem. Environ., 19, 57-66.

Barnes, N. M. Khodse, V. B. Lotlikar, N. P. Meena, R. M. \& Damare, S. R. (2018). Bioremediation potential of hydrocarbon-utilizing fungi from select marine niches of India. 3 Biotech, 8, 1-21. DOI: 10.1007/s13205-017-1043-8.

Barragán-Huerta, B. E., Costa-Pérez, C., Peralta-Cruz, J., Barrera-Cortés, J., Esparza-García, F. \& RodríguezVázquez, R. (2007). Biodegradation of organochlorine pesticides by bacteria grown in microniches of the porous structure of green bean coffee. International Biodeterioration \& Biodegradation, 59 (3), 239-244. DOI. org/10.1016/j.ibiod.2006.11.001.

Biswas, K. (2015). Biological agents of bioremediation: a concise review. Frontiers in Environmental Microbiology, 1 (3), 39-43. DOI: 10.11648/j.fem.20150103.11.

Botero, L. R., Mougin, C., Peñuela, G. \& Barriuso, E. (2017). Formation of 2, 4-D bound residues in soils: New insights into microbial metabolism. Science of the Total Environment, 584, 715-722. DOI.org/10.1016/j.scitotenv.2017.01.105.

Brenner, D. J., Krieg, N. R., Staley, J. T. \& Garrity, G. M. (2005). Bergey's Manual of Systematic Bacteriology, 2nd. 2.

Chakrabarty, A. M. (2017). Biodegradation and Detoxification of Environmental Pollutants. CRC Press. 31 p.

Chaudhry, G. R. \& Huang, G. H. (1988). Isolation and characterization of a new plasmid from a Flavobacterium sp. which carries the genes for degradation of 2 , 4-dichlorophenoxyacetate. Journal of bacteriology, 170 (9), 3897-3902. DOI: 10.1128/jb.170.9.3897-3902.1988.

Chen, S., Yang, L., Hu, M. \& Liu, J. (2011). Biodegradation of fenvalerate and 3-phenoxybenzoic acid by a novel Stenotrophomonas sp. strain ZS-S-01 and its use in bioremediation of contaminated soils. Applied microbiology and biotechnology, 90 (2), 755-767. DOI: 10.1007/s00253010-3035-z.

Deng, S., Chen, Y., Wang, D., Shi, T., Wu, X., Ma, X. \& Li, Q. X. (2015). Rapid biodegradation of organophosphorus pesticides by Stenotrophomonas sp. G1. Journal of hazardous materials, 297, 17-24. DOI: 10.1016/j. jhazmat.2015.04.052.

Donnelly, P. K., Entry, J. A. \& Crawford, D. L. (1993). Degradation of atrazine and 2, 4-dichlorophenoxyacetic acid by mycorrhizal fungi at three nitrogen concentrations in vitro. Appl. Environ. Microbiol., 59 (8), 2642-2647. DOI: 10.1080/03601230701735227.

Drouin, P., Sellami, M., Prevost, D., Fortin, J. \& Antoun, H. (2010). Tolerance to agricultural pesticides of strains belonging to four genera of Rhizobiaceae. Journal of Environmental Science and Health Part B, 45(8), 757-765. DOI.org/10.1080/03601234.2010.515168.

Dubey, K. K. \& Fulekar, M. H. (2012). Chlorpyrifos bioremediation in Pennisetum rhizosphere by a novel potential degrader Stenotrophomonas maltophilia MHF ENV20. World Journal of Microbiology and Biotechnology, 28(4), 1715-1725. DOI: 10.1007/s11274011-0982-1.

Feng, F., Ge, J., Li, Y., He, S., Zhong, J., Liu, X. \& Yu, X. (2017). Enhanced degradation of chlorpyrifos in rice (Oryza sativa L.) by five strains of endophytic bacteria and their plant growth promotional ability. Chemosphere, 184, 505-513. DOI: 10.1016/j.chemosphere.2017.05.178.

Harms, H., Schlosser, D. \& Wick, L. Y. (2011). Untapped potential: exploiting fungi in bioremediation of hazardous chemicals. Nature Reviews Microbiology, 9 (3), 177. DOI:10.1038/nrmicro2519.

Huang, Y., Zhao, X., \& Luan, S. (2007). Uptake and biodegradation of DDT by 4 ectomycorrhizal fungi. Science of the Total Environment, 385(1-3): 235-241. DOI: 10.1016/j.scitotenv.2007.04.023.

Ishag, A.E. S.A.,Abdelbagi,A. O., Hammad,A. M.A., Elsheikh, E. A. E., Elsaid, O. E. \& Hur, J. H. (2017). Biodegradation of endosulfan and pendimethalin by three strains of bacteria isolated from pesticides-polluted soils in the Sudan. Applied Biological Chemistry, 60 (3), 287-297. DOI.org/10.1007/ s13765-017-0281-0.

Islas-Pelcastre, M., Villagómez-Ibarra, J. R., MadariagaNavarrete, A., Castros-Rosas, J., González-Ramírez C. A. \& Acevedo-Sandoval, O. A. (2013). Bioremediation perspectives using autoch thonous speceies of Trichoderma sp. For degradation of atrazine in agricultural soil from 
the Tulancingo Valley, Hidalgo, Mexico. Tropical and Subtropical Agroecosystems, 16(2). 265-276.

Mao, H., Liu, H., Gao, Z., Su, T. \& Wang, Z. (2015). Biodegradation of poly (butylene succinate) by Fusarium sp. FS1301 and purification and characterization of poly (butylene succinate) depolymerase. Polymer degradation and stability, 114, 1-7. DOI.org/10.1016/j. polymdegradstab.2015.01.025.

Maheshwari, R., Singh, U., Singh, P., Singh, N., Lal, B. \& Rani, B. (2014). To decontaminate wastewater employing bioremediation technologies. Journal of Advanced Scientific Research, 5 (2): 7-15.

Meharg, A. A., Cairney, J. W. \& Maguire, N. (1997). Mineralization of 2, 4-dichlorophenol by ectomycorrhizal fungi in axenic culture and in symbiosis with pine. Chemosphere, 34 (12), 2495-2504. DOI.org/10.1016/ S0045-6535(97)00005-2.

Mirgain, I., Green, G. A. \& Monteil, H. (1993). Degradation of atrazine in laboratory microcosms: isolation and identification of the biodegrading bacteria. Environmental Toxicology and Chemistry: An International Journal, 12 (9), 1627-1634. DOI.org/10.1002/etc.5620120911.

Mohiddin, F. A. \& Khan, M. R. (2013). Tolerance of fungal and bacterial biocontrol agents to six pesticides commonly used in the control of soil borne plant pathogens. African Journal of Agricultural, 8(43), 5272-5275. DOI: 10.5897/ AJAR11.677.

Mori, T., Wang, J., Tanaka, Y., Nagai, K., Kawagishi, H. \& Hirai, H. (2017). Bioremediation of the neonicotinoid insecticide clothianidin by the white-rot fungus Phanerochaete sordida. Journal of hazardous materials, 321, 586-590. DOI: 10.1016/j.jhazmat.2016.09.049.

Motosugi, K. \& Soda, K. (1983). Microbial degradation of synthetic organochlorine compounds. Cellular and Molecular Life Sciences, 39 (11), 1214-1220. DOI: 10.1007/ BF01990358.

Mousawi, A. N. M. (2005). Study of bacterial resistance to organophosphorous pesticides in Iran. Journal of Environmental Health Science \& Engineering, 2 (3), 207-211.

Nykiel-Szymańska, J., Stolarek, P. \& Bernat, P. (2018). Elimination and detoxification of 2, 4-D by Umbelopsis isabellina with the involvement of cytochrome P450. Environmental Science and Pollution Research, 25 (3), 2738-2743. DOI: 10.1007/s11356-017-0571-4.

Ortíz, I., Velasco, A., Le Borgne, S. \& Revah, S. (2013). Biodegradation of DDT by stimulation of indigenous microbial populations in soil with cosubstrates. Biodegradation, 24 (2), 215-225. DOI: 10.1007/s10532012-9578-1.

Ortiz-Hernández, M. L., Monterrosas-Brisson, M., Yanez-Ocampo, G. \& Sánchez-Salinas, E. (2001). Biodegradation of methyl-parathion by bacteria isolated of agricultural soil. Revista Internacional de
Contaminación Ambiental, 17 (3), 147-155.

Ortiz-Hernández, M. L., Sánchez-Salinas, E., Olvera-Velona,A. \& Folch-Mallol, J. L. (2011). Pesticides in the environment: impacts and its biodegradation as a strategy for residues treatment. In Pesticides-formulations, effects, fate. Intech. Open, 551-574. DOI: 10.5772/1004.

Ortiz-Hernández, M. L. \& Sánchez-Salinas, E. (2010). Biodegradation of the organophosphate pesticide tetrachlorvinphos by bacteria isolated from agricultural soils in México. Revista internacional de contaminación ambiental, 26 (1), 27-38.

Panek, J. \& Frąc, M. (2018). Development of a qPCR assay for the detection of heat-resistant Talaromyce sflavus. International journal of food microbiology, 270, 44-51. DOI.org/10.1016/j.ijfoodmicro.2018.02.010.

Peter, L., Gajendiran, A., Mani, D., Nagaraj, S. \& Abraham, J. (2015). Mineralization of malathion by Fusarium oxysporum strain JASA1 isolated from sugarcane fields. Environmental Progress \& Sustainable Energy, 34 (1), 112-116. DOI: 10.1002/ep.11970.

Potin, O., Rafin, C. \& Veignie, E. (2004). Bioremediation of an aged polycyclic aromatic hydrocarbons (PAHs)contaminated soil by filamentous fungi isolated from the soil. International Biodeterioration \& Biodegradation, 54 (1), 45-52. DOI: 10.1016/j.ibiod.2004.01.003.

Reader, U. \& Broda, P. (1985). Rapid preparation of DNA from filamentous fungi. Letter Applied Microbiology, 1, 17-20. DOI.org/10.1111/j.1472-765X.1985.tb01479.x.

Robles-González, I., Ríos-Leal, E., Ferrera-Cerrato, R., EsparzaGarcía, F., Rinderkenecht-Seijas, N. \& Poggi-Varaldo, H. M. (2006). Bioremediation of a mineral soil with high contents of clay and organic matter contaminated with herbicide 2, 4-dichlorophenoxyacetic acid using slurry bioreactors: effect of electron acceptor and supplementation with an organic carbon source. Process Biochemistry, 41 (9), 1951-1960. DOI.org/10.1016/j.procbio.2006.04.004.

Saafan, A. E., Azmy, A. F., Amin, M. A., Ahmed, S. H. \& Essam, T. M. (2016). Isolation and characterization of two malathion degrading Pseudomonas sp. in Egypt. African Journal of Biotechnology, 15 (31), 1661-1672. DOI: 10.5897/AJB2016.15273.

Sethunathan, N. \& Yoshida, T. (1973). A Flavobacterium sp. that degrades diazinonand parathion. Canadian Journal of Microbiology, 19 (7), 873-875. DOI: 10.1139/m73-138.

Shafiani, S. \& Malik, A. (2003). Tolerance of pesticides and antibiotic resistance in bacteria isolated from wastewater-irrigated soil. World Journal of Microbiology and Biotechnology, 19 (9), 897-901. DOI.org/10.1023/ B:WIBI.0000007290.94694.4f.

Shukla, K. P., Singh, N. K., \& Sharma, S. (2010). Bioremediation: developments, current practices and perspectives. Genet. Eng. Biotechnol. J., 3: 1-20.

Singh, P., Suri, C. R. \& Cameotra, S. S. (2004). Isolation of a member of Acinetobacter species involved in atrazine 
degradation. Biochemical and biophysical research communications, 317 (3), 697-702. DOI: 10.1016/j. bbrc.2004.03.112.

Smejkal, C. W., Seymour, F. A., Burton, S. K. \& LappinScott, H. M. (2003). Characterization of bacterial cultures enriched on the chlorophenoxyalkanoic acid herbicides 4-(2, 4-dichlorophenoxy) butyric acid and 4-(4-chloro2-methylphenoxy) butyric acid. Journal of Industrial Microbiology and Biotechnology, 30 (9), 561-567. DOI: 10.1007/s10295-003-0086-5.

Wang, B., Liu, W., Liu, X., Franks, A. E. Teng, Y. \& Luo, Y. (2017). Comparative analysis of microbial communities during enrichment and isolation ofDDT-degrading bacteria by culture-dependent and-independent methods. Science of the Total Environment, 590, 297-303. DOI: 10.1016/j. scitotenv.2017.03.004.

Wei, S., Xue-na, Z., Hai-bin, J., Sheng-dong, F., Zhin-xin, Y., Ou-ya,Z., Yu-ling, L. (2017). Effective remediation of aged HMW-PAHs polluted agricultural soil by the combination of Fusarium sp. And smooth bromegrass (Bromus inermis leyss). Journal of integratiev agriculture, 16(1). 199-209. DOI: 10.1016/S2095-3119(16)61373-4.

Weisburg, W., Barns, S. M., Pelletier, D. A. \& Lane, D. J. (1991). 16S Ribosomal DNA amplification for phylogenetic study. Journal of Bacteriology, 173(2). 697-703. DOI: 10.1128/ jb.173.2.697-703.1991.
White, T. J., Bruns, T., Lee, S. \& Taylor, J. (1990). Amplification and direct sequencing of fungal ribosomal RNA genes for phylogenetics. PCR Protocols: A Guide to Methods and Applications, Academic Press, New York, 315-322. DOI:10.1016/b978-0-12-372180-8.50042-1.

Xia, Z. Y., Zhang, L., Zhao, Y., Yan, X., Li, S. P., Gu, T. \& Jiang, J. D. (2017). Biodegradation of the herbicide 2, 4-dichlorophenoxyacetic acid by a new isolated strain of Achromobacter sp. LZ35. Current microbiology, 74 (2), 193-202. DOI: 10.1007/s00284-016-1173-y.

Yadav, J. S. \& Reddy, C. A. (1993). Mineralization of 2, 4-dichlorophenoxyacetic acid (2, 4-D) and mixtures of 2, 4-D and 2, 4, 5-trichlorophenoxyacetic acid by Phanerochaete chrysosporium. Appl. Environ. Microbiol., 59 (9), 29042908. DOI: 10.1128/AEM.59.9.2904-2908.1993.

Yamashita, S., Nakagawa, H., Sakaguchi, T., Arima, T. H. \& Kikoku, Y.(2018). Design of a species-specific PCR method for the detection of the heat-resistant fungi Talaromyces macrospores and Talaromyces trachyspermus. Letters in applied microbiology, 66 (1), 86-92. DOI: 10.1111/ lam. 12818.

Yao, L., Jia, X., Zhao, J., Cao, Q., Xie, X., Yu, L. \& Tao, Q. (2015). Degradation of the herbicide dicamba by two sphingomonads via different O-demethylation mechanisms. International Biodeterioration \& Biodegradation, 104, 324-332. DOI.org/10.1016/j.ibiod.2015.06.016. 